\title{
Processing Big Data in streaming for fault prediction: an industrial application
}

\author{
Angelo Corallo \\ Dipartimento di Ingegneria \\ dell'Innovazione \\ Università del Salento \\ Lecce, Italy \\ angelo.corallo@unisalento.it \\ Mariangela Lazoi \\ Dipartimento di Ingegneria \\ dell'Innovazione \\ Università del Salento \\ Lecce, Italy \\ mariangela.lazoi@unisalento.it
}

\author{
Annamaria Crespino \\ Dipartimento di Ingegneria \\ dell'Innovazione \\ Università del Salento \\ Lecce, Italy \\ annamaria.crespino@unisalento.it \\ Marianna Lezzi \\ Dipartimento di Ingegneria \\ dell'Innovazione \\ Università del Salento \\ Lecce, Italy \\ marianna.lezzi@unisalento.it
}

\author{
Carla DiBiccari \\ Dipartimento di Ingegneria \\ dell'Innovazione \\ Università del Salento \\ Lecce, Italy \\ carla.dibiccari@unisalento.it
}

\begin{abstract}
In the current data-driven industrial scenario, Big Data processing plays a leading role in enhancing business performance. An ever increasing number of working machines are equipped with smart devices (such as sensors and actuators) which are in charge of monitoring machine status in real time and implement corrective actions before the workpiece quality is compromised or machine is damaged. However, many manufacturing companies do not take advantage of the use of Big Data coming from their production systems. In some cases, Big Data analytics is an un-explored issue since it is considered time and resources consuming. Moreover, the real benefits of processing, in real time, industrial data are usually underestimated. The European project TOREADOR wants to extend and facilitate the diffusion of Big Data analytics within industrial contexts, in order to generate greater value for companies. Focusing on the aerospace components manufacturing process (as one of the case studies of the Project), the paper aims to describe the main developments and lessons learned by the customization of TOREADOR Big Data analytics platform to process and analyze data from $\mathrm{CNC}$ machines sensors.
\end{abstract}

Keywords - big data, fault detection, aerospace industry, Toreador project

\section{INTRODUCTION}

A huge volume and variety of data is currently generated within digital and connected aeronautical supply chain [1]. Although the volume of data associated with industrial processes and products is growing exponentially, the aeronautical companies currently leverage only a small part of it; still, in many cases, real-time data processing and analysis are not conducted yet. In such a data-driven industrial context, Big Data analytics promises significant performance improvements along the entire supply chain [2]. In particular, taking advantage of manufacturing data, in real time, means to carry out predictive maintenance able to improve quality, efficiency and effectiveness of manufacturing operations, as well as influence the productivity and profitability of the entire manufacturing process [3].
In line with the principles of the Digital Agenda for Europe [4], an important Italian aerospace company, which designs, manufactures and maintains components and systems for civil and military aviation, has committed to the smart use of Information and Communication Technologies (ICT) with the aim to better leverage its manufacturing data. Today, in the majority of the Company's manufacturing cells, the Computer Numerical Control (CNC) machines may record a large amount of data from sensors on machine health status and working parameters. The challenge is to gather and analyze such data in order to obtain useful insights for better maintenance decision making, thus improving performance of the manufacturing lines.

In this context, the TOREADOR (TrustwOrthy modelawaRE Analytics Data platfORm) project, funded by the call H2020-ICT-2015, aims to overcome some major hurdles that until now have prevented many European companies from reaping the full benefits of Big Data analytics (BDA). Many companies and organizations in Europe have become aware of the potential competitive advantage they could get by timely and accurate Big Data analytics, but lack the IT expertise and budget to fully exploit BDA. In order to overcome this hurdle, TOREADOR adopts a Model-based BDA-as-a-Service (MBDAaaS) approach, providing a user friendly and customizable platform able to manage the entire Big Data analysis process and its artefacts.

Based on the research activities carried out in TOREADOR, the paper aims to describe the lessons learned from the customization of the TOREADOR platform in an aerospace manufacturing context, by applying Model-based Big Data Analytics as-a-Service (MBDAaaS) framework [5].

The next section of the paper provides an overview on the predictive maintenance role in the manufacturing industry. Section III describes the specific aerospace context on which the TOREADOR platform was customized; while, section IV details the realized application. The final sections, concerning lessons learned and conclusions, end the paper. 


\section{RELATED SCIENTIFIC STUDIES}

Recent studies estimate that the unplanned downtime costs for the manufacturing industry are $\$ 50$ billion per year [6]. The use of predictive maintenance and failure detection can support the sector for reducing its costs and improving companies' competitive advantage.

Predictive maintenance (PdM) is currently enabled by smart technologies, usually embedded in the physical assets. In particular, by leveraging the massive amount of data produced by these technologies and applying the right Big Data analytics, machine failures can be predicted, with evident benefits in terms of parts' manufacturing efficiency and unnecessary downtime minimization. Furthermore, early failure detection is well accepted in the industry, since it can potentially eliminate catastrophic machine failures [7]. Many anomaly detection techniques have already been developed in machine learning and statistical domains by applying supervised, semi-supervised and unsupervised techniques [8]. Although the majority of them were designed for generic application domains, recent research has also focused on techniques developed for specific ones. In particular, the problem of detecting manufacturing anomalies has received little attention [9]. Several algorithms are widely used today to identify the abnormal behavior of a subsystem, such as: Principle Component Analysis (PCA), K-Means, C-Means, Hierarchical clustering, neural networks, Gaussian Mixture Model (GMM), and Modified Rank Order Clustering (MROC) [7]. The main challenge remains, perhaps, to choose the most fitting algorithm in relation to the industrial context and the business goals, as well as the appropriate approach to implement it considering the pre-existing technological infrastructure.

\section{RESEARCH AND INDUSTRIAL CONTEXT}

TOREADOR project has four pilot cases for which develop and test all the methodological and technological solutions of the project. One of this is the aerospace pilot, managed by DTA (Apulian District of Aerospace) together with Università del Salento. For this pilot, three different use cases have been identified:

- In-process predictive analytics on workpieces dimensions, which concerns the analysis of work-cycles in the different production cells to predict potential product anomalies;

- Root cause analysis of quality notifications in the inspection phase, regarding the investigation, during the inspection phase, of the root cause that has generated the anomaly on product;

- Fault Prediction, which concerns the analysis of data collected from both the work cycle in the production cells and the inspection activities, in order to predict machine faults before the quality of the product is jeopardised.

The focus of this paper is on the use case of fault prediction that have required a more customized and deeper development.

The aim of the fault prediction use case is the detection and prediction of possible machines' faults, which may happen during the work-cycle. Specifically the goal is to identify the thresholds associated with different faults occurring on machines. In order to achieve this goal, machine health status should be monitored, by collecting the working data of machines involved in the work-cycle and identifying the range of values, out of which, the alert may be raised. In this case, the data analysis is run in near realtime and the dataset is continuously updated in append with data produced during the work-cycle.

The first step to deploy fault detection analytics into Toreador platform consists of defining the case study perimeter. Based on the aerospace pilot business needs, the manufacturing cell of bevel gears has been selected. The Bevel Gear Cell presents two processing sub-cells in a sequential order (one before and the other after the Heat Treatment (HT) of the parts) and a sub-cell for final inspection of the produced components. Three lathes of the cell were considered for the pilot. The machines are connected in the network through sensors, which collect the machine working data, e.g. temperature, angular velocity, spindle speed, etc.

Modern CNC machines, like those of the bevel gear cell, are able to recognize a pre-determined set of failure states and are provided with servomechanisms that cause the machine to provide a diagnostic code on the controller screen, adjust its behavior or even stop completely the manufacturing process whenever a failure state is detected.

The goal is to recognize the pattern of the machine behavior that leads to each failure and signal it to users in good time to take decisions that will prevent quality from being jeopardized. The main problems to be handled involve: learning a data classification model, identifying a correct definition of faults and discovering a suitable model for prediction.

In this use case, the aerospace company is willing to go beyond $\mathrm{CNC}$ manufacturer pre-set failure states. Specifically, it would identify unknown machine parameter ranges of values, which allow us to detect peculiar failure states that were not pre-determined by the manufacturer, but can damage the machine and affect the workpiece quality as well. The recognition of the behavioral pattern (in terms of parameters' values) that precedes each single faulty state closes the use case allowing prediction.

Therefore, the use case is comprised of the following steps: 
- Detection and Prediction phase for new faults;

- Description and Prediction for pre-determined faults.

It is noteworthy that detection of customized faults can be based on thresholds defined by expert technicians, as a consequence of the fact that the analytics algorithm for fault detection often performs a descriptive task, based on the dataset provided by the domain engineer, which aims at building a diagnosis model to detect fault.

\section{THE REALIZED APPLICATION}

Following the MDA for developing the MBDAaaS framework, the three models (i.e. CIM, PIM and PSD), representing the fault prediction use case are provided as also described in [10].

The first step produced the .json file containing the specifications for the CIM model (declarative one) in terms of goals with indicators and objectives (see Fig. 1). For example, the analytics for the discovery of the behavioral model of machines (an unsupervised, machine learning clustering) has been defined by three Goals: G1 (Task, clustering), G2 (Models, Descriptive), G3 (Learning Approach, Unsupervised).

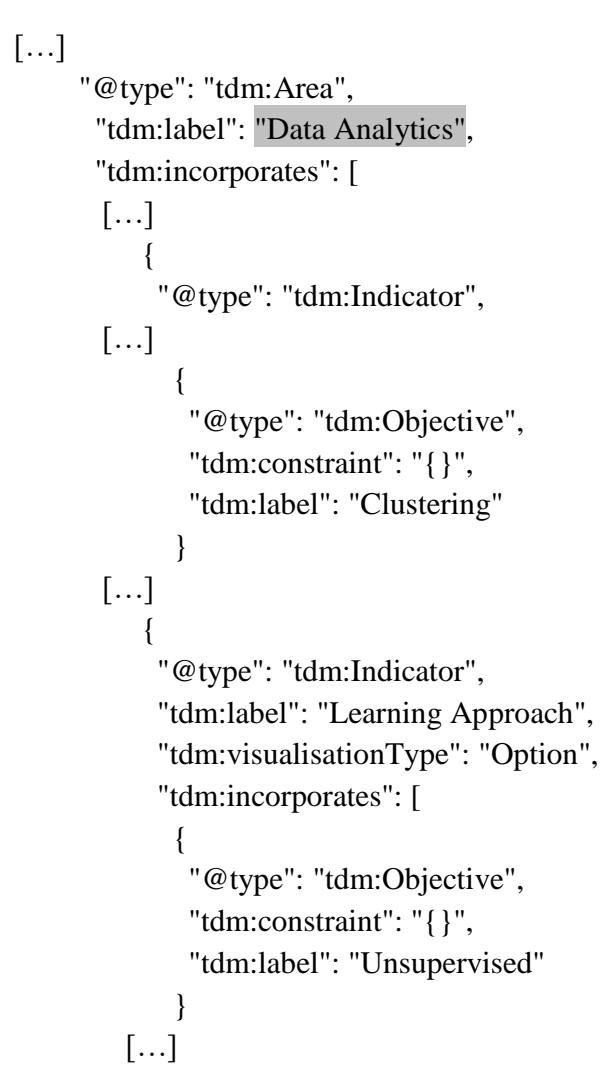

Fig. 1. A fragment of the declarative model in JSON for the Fault Prediction Process.

The second step concerned the PIM (or procedural model) definition, describing in the OWL-S workflow how analytics should be executed and parallelized following the requirements and constraints specified in the declarative model.

The third step produced the PSM, i.e. the deployment model, which maps each operation and tool to specific services. The deployment model has been designed starting from the company on-premises infrastructure for data processing, orchestration, analytics and visualization. The transformation from procedural model to deployment model is based on the compiler that takes as input the OWL-S workflow file and information on the target platform and produces as output a technology dependent workflow.

The choice of OWL language was due to the fact that it supports automatic Web service discovery, invocation, composition, and interoperation. The OWL-S ontology, instead, is structured in three interrelated sub-ontologies: profile, process model, and grounding. The profile ontology is used to express what "does" the service; the process model describes "how it works"; and the grounding maps the constructs of the process model onto detailed specifications of message formats, protocols, and so forth. In addition, in order to specify the service by using the Toreador methodology, OWL-S was extended to define a mapping with the goals of the declarative model, used for the service selection phase. In this way, the proposed model assumes the construction of complex services as a composition within a process model.

\section{A. Architecture}

An instance of Toreador platform was configured on the company Amazon Web Services (AWS), by adopting the Kubernetes cluster manager (see Fig. 2). As an evidence, Toreador platform is based on the deployment of microservices units on a cluster infrastructure. The Kubernetes is a cluster orchestrator that allows Docker based services to share the cluster resources and run on a distributed environment. The cluster infrastructure includes three workers and one master with the following components:

- Pods for single-machine instances like Redis, Mysql, Spring Dataflow server, Service Catalogue;

- Deployments for scaled stateless replicas like Spring Cloud Dataflow (SCDF), Spark, HDFS, Kafka, Nexus Server and Viz;

- Stateful sets for stateful replicas where unique network identifier and stable storage is needed, like Cassandra or Spark/HDFS nodes.

Spring Cloud Dataflow (SCDF) is the selected engine that allows users to define and run an application data flow on a message bus (Kafka). The Toreador platform exposes an API to compose data flows between batch and streaming 


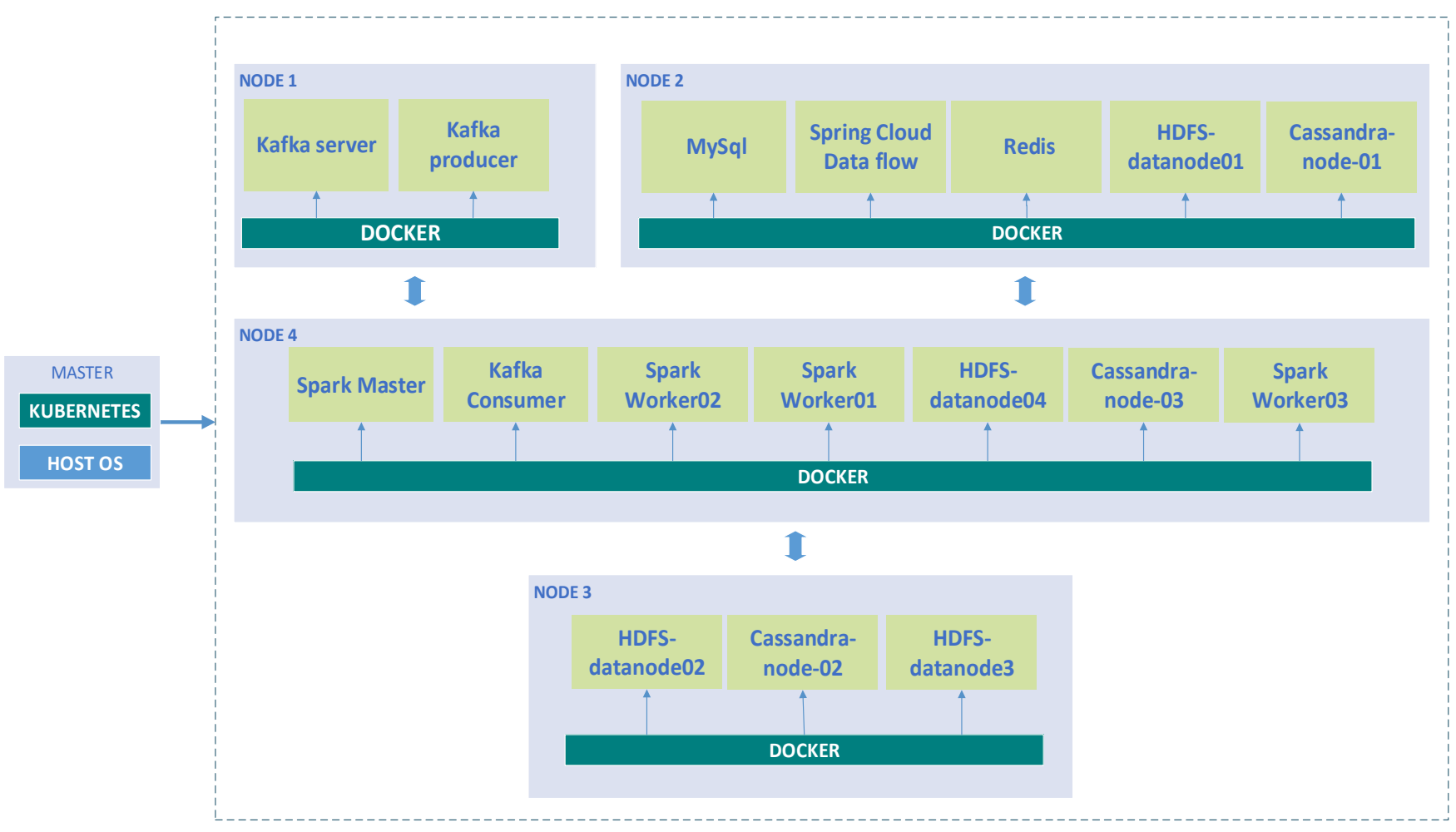

Fig. 2. Toreador Platform instanced on AWS

analytics services. The Service Catalogue deployed as Kubernetes Pod consists of a Catalogue-API (REST interface for the services catalogue) and a MySql data base and world state; in other words, information production is described by the inputs and outputs of the process. All services developed for the Toreador Big Data Analytics are

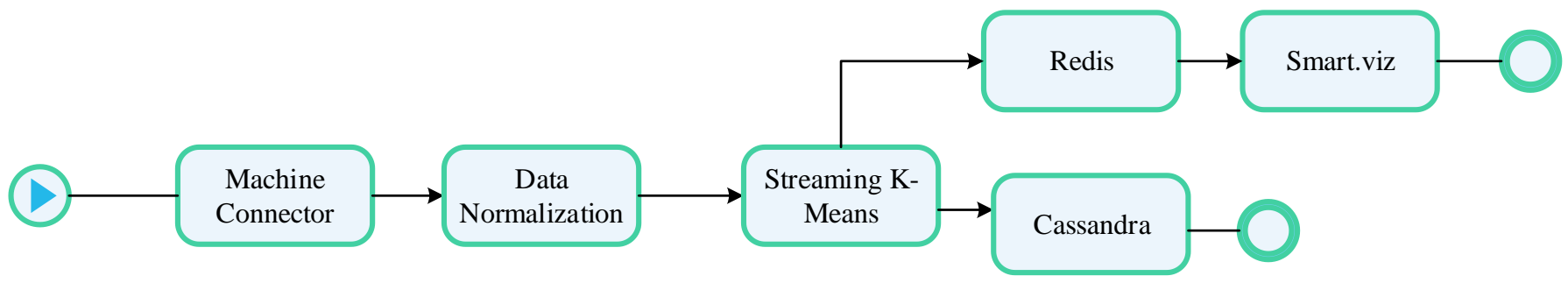

Fig. 3. Fault Prediction data processing pipeline

to store services definitions. The Nexus Server has been used as repository manager to host the Docker images. Finally, a visualization framework (Viz) has been deployed to report the analytics graphical results.

\section{B. Data processing pipeline}

In line with the aims of the Toreador methodology [5], the fault prediction data processing pipeline is a composition of a series of simple "atomic process". An atomic process is a description of a service that expects one message and returns one message in response. On the other hand, a composite process is one that maintains some state and may have several distinct purposes: acquisition or transformation of data or knowledge; modification of the state of objects or services within the service environment; and finally, application of changes within the real world. A service can generate and return some new information based on input included in a Toreador service catalogue. The compiler retrieves those services that are compatible with the goals and constraints specified in the declarative model. In particular, the compiler receives its OWL-S composition, serialized in RDF/XML format, automatically parses this specification and thus generate a new file specifying the workflow based on the language accepted by SCDF.

Fig. 3 shows the fault prediction data processing pipeline based on six services. These services were selected following the Toreador methodology.

The "Machine Connector" is the first service of the workflow execution. It reads the stream of sensor data that measure various parameters of the production process, such as spindle speed, motor load, motor power and path federate (Fig. 4 shows a fragment of the OPC-UA Client Java 
package that implements a connection to collect sensor data). The data set also includes meta-data about the machine and measurement data. Meta-data includes information about the machine type and number of sensors per machine. Each measurement is timestamped and can be of different types: double precision, integer, string. Table 1 shows an extract of the values transmitted by a specific sensor.

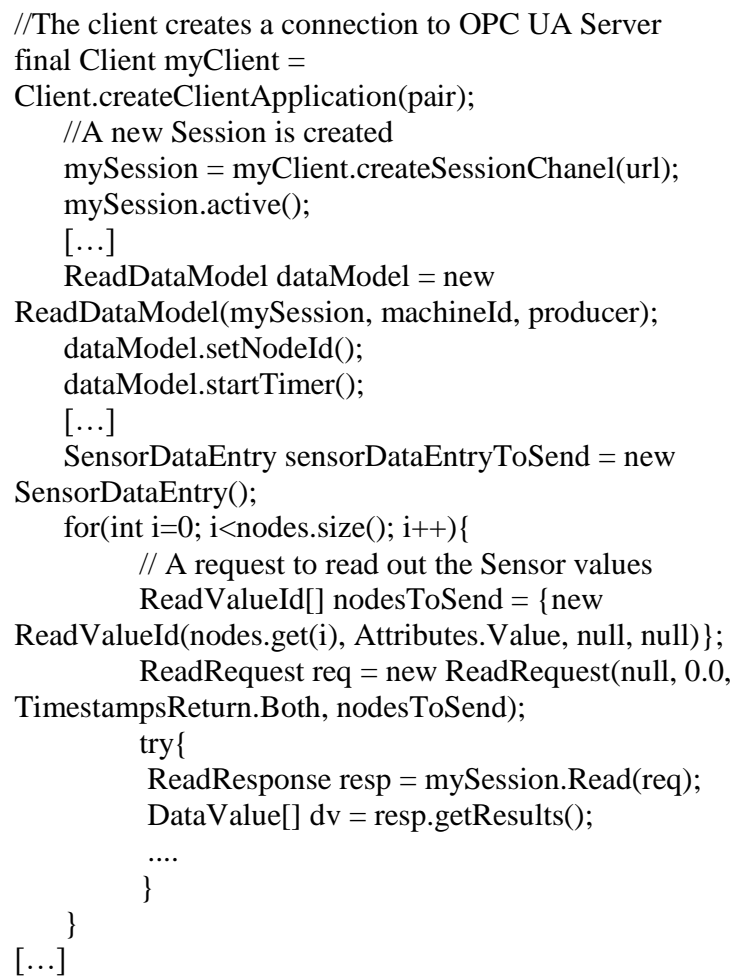

Fig. 4. A fragment of the Client connection to read the sensor data.

Processing data to extract business benefit in real time is crucial, it may require machine-learning models or calculate simple operations, and in any case, it should be a fast and scalable process to ensure system availability. The first step clusters the elements using the "Streaming K-Means" service; it has been deployed to execute a K-Means algorithm. This service has to maintain $K$ cluster centers, where the number $K$ is defined in the meta-data for each program status of each individual machine. All data items from sensors are used to compute the cluster centroids. In order to find anomalies, for each data window, it is considered that if the sequence of last $\mathrm{N}$ data points has a combined transition probability lower than a threshold an alert about the machine is raised. When an anomaly is detected, the first time stamp amongst the last $\mathrm{N}$ data points is output as an alert that an anomaly has been detected at that instant of time.

Among all available real-time data storage services, "Cassandra" was selected to enable the ingestion of temporal data in real time and maintain these records with a long retention period; while "Redis" service was chosen to achieve a pure in-memory ingestion of key-value records and to support the analysis report executed by "Smart.Viz" service.

In particular, the visualization process, delegated to "Smart.viz", leads to the generation of charts in 5-step: data source and database selection; database query, data model specification; mapping; chart tuning and visualization.

"Redis" was used as data source in memory because of it offers response times in milliseconds, allowing the creation of applications that manage millions of requests per second in real time. The Redis instance is selected to keep all the volumes of data related to analysis results on the fault prediction process.

TABLE I. AN EXTRACT OF THE SENSOR VALUES

\begin{tabular}{|l|l|}
\hline Time & X1_Motor_Power \\
\hline 2018-05-29 12:41:18 & 56.263 \\
2018-05-29 12:41:25 & 56.263 \\
$2018-05-2912: 41: 33$ & 56.263 \\
$2018-05-2912: 41: 41$ & 56.263 \\
$2018-05-2912: 41: 49$ & 56.263 \\
$2018-05-2912: 41: 57$ & 0.037 \\
$2018-05-2912: 42: 05$ & 0.037 \\
$2018-05-2912: 42: 13$ & 0.037 \\
$2018-05-2912: 42: 21$ & 0.037 \\
$2018-05-2912: 42: 28$ & 0.037 \\
$2018-05-2912: 42: 36$ & -0.008 \\
$2018-05-2912: 42: 44$ & -0.008 \\
$2018-05-2912: 42: 52$ & -0.008 \\
$2018-05-2912: 43: 00$ & -0.038 \\
$2018-05-2912: 43: 08$ & -0.038 \\
$2018-05-2912: 43: 16$ & -0.038 \\
$\ldots \ldots \ldots \ldots \ldots \ldots \ldots \ldots \ldots$ & $\ldots \ldots \ldots \ldots$ \\
\hline
\end{tabular}

\section{LESSON LEARNED}

The goal of the study was to implement within a complex industrial context, such as aerospace components manufacturing, a Big Data analytics, able to process the streaming data flow from $\mathrm{CNC}$ machines with the aim to visualize in real time the machine working status and predict possible machine faults. In particular, the implementation was realized following the three-step methodology proposed by Toreador project. In the following, a short list of the main difficulties that have been faced:

- Configuration of analytics according with the model-based BDA-as-a-service (MBDAaaS) Toreador approach;

- Configuration and installation of the Toreador platform on the company network, due to confidentiality of aerospace data to be managed; 
- Development and integration of Big Data analytics services within the Toreador platform.

In facing these challenges, the study of the industrial domain, in terms of technological assets and physical processes, has proven to be necessary to build an analytic system able to process industrial data in real time. As an evidence, the system developed for the aerospace study case has required knowledge about:

- Sensors installed on the working machines, which produce streaming data;

- Industrial communication protocols (such as OPC-UA), in charge of sending such data from sensors to servers;

- OPC-enabled applications to be configured in order to leverage sensor data by using Big Data analytics.

Moreover, another relevant point for the successful implementation of Big Data analytics in the aerospace context was the active collaboration with the other partners of the project. All collaborations were crucial to define and specify the architecture of the TOREADOR platform with the aim at eliciting the technical and functional requirements.

The TOREADOR platform architecture was defined to provide a big data analytics as service, by integrating and exploiting the toolsets and services for managing and analyzing big data value in several domains, such as the Aerospace one. On the basis of the architectural models, the services and toolsets were integrated on this platform to allow the users to design data processing and analytics workflows, and to configure the specifications and services. A goal-oriented approach, where managers can capture the business process through goals and sub-goals, observe processes execution, catch anomalies, and take appropriate actions, has proven to be useful. In particular, the platform allowed the aerospace use case to:

- Gather and integrate Big Data related to manufacturing processes;

- Apply predictive analytics techniques for machinery performance optimization;

- Report to company staff the analytics results in order to improve the decision making process.

Technical and performance requirements were collected for the deployment of TOREADOR system in reference to a manufacturing process able to produce data in (nearly) realtime. In collaboration with the other experts of the TOREADOR project, the right algorithms, data analytics techniques, and data reporting have been chosen to manage complex manufacturing behavior. In particular, the identification of appropriate technologies to recover data from sensors along the machinery work-cycle was revealed to be necessary.

Another lesson learned from the development phase concerns the ever-growing volume of sensor data, which needs to be managed by different technologies than the traditional ones. In particular, the traditional data modeling has been discarded as no logger applicable in the aerospace industrial scenario and a mix of technologies, going from NoSQL ("notonlySQL") databases (i.e. Cassandra or Hive), distributed parallel computing systems (i.e. Hdoop), and cluster manager systems (i.e. Kubernetes), have been shown to be useful.

\section{CONCLUSIONS}

The paper describes the developments and lesson learned based on the application of the MBDAaaS methodology proposed in the TOREADOR project for leading big data solutions in an aerospace manufacturing company domain. The proposed methodology is based on the model driven engineering approach. It lies on a data-driven dedicated architecture with semantic properties to handle the big amount of data. This allows us to simplify the chain of competences needed to manage the whole big data pipeline and support the analytics task.

Among three possible use cases (i.e. In-process predictive analytics on workpieces dimensions, Root cause analysis of quality notifications in the inspection phase and Fault Prediction) the paper is focalized on the fault prediction for the analysis of data collected in a bevel gear cell where there are a number of modern CNC machines. These ones are able to recognize a pre-determined set of failure states and adjust their behavior. With the solution developed in TOREADOR, it is aimed to go beyond CNC manufacturer pre-set failure states.

In this context, a three models (i.e. CIM, PIM and PSD) of the MBDAaaS methodology are applied for representing the fault prediction use case. Furthermore, given the specific and critical features of an aerospace domain, several difficulties have been managed about: configuration of analytics according with the model-based BDA-as-a-service (MBDAaaS) TOREADOR approach; configuration and installation of the TOREADOR platform on the company network; and development and integration of services within the TOREADOR platform. Technical issues and lesson learned are described in the paper to lead future developments and the replication of similar application in others aerospace companies and also, in others complex industrial sectors.

In general, the Toreador framework for Model-based Big Data Analytics-as-a-Service has proved to be a useful mean to manage real-time data analytics and deploy a workflow that addresses specific analytics goals. As a matter of fact, the Toreador platform configurations provides all necessary computational components and technological resources necessary to apply the analytics for streaming sensors data. 
There are several improvements generated by the implementation of this pilot and the interaction with a powerful MBDAaaS tool, such as the implementation of data collection and stream in real-time from a set of CNC machines, by applying different agents to standardize the stream according to the OPC-UA communication protocol, and the agnostic description of the single atomic services in the use case implementation, which allows to easy re-use them in new different scenarios.

Future researches will be based on the application and test on the pilot site also of others type of analytics and services for big data management rising from $\mathrm{CNC}$ machines.

\section{ACKNOWLEDGMENT}

This project has received funding from the European Union's Horizon 2020 research and innovation program under the TOREADOR project, grant agreement $n^{\circ} 688797$.

\section{REFERENCES}

[1] T. Armes, M. Refern, "Using Big Data and Predictive Machine Learning in Aerospace Test Environments", 2013 IEEE AUTOTESTCON, Schaumburg, IL, USA, pp.1-5, 2013.

[2] S. Tiwari, H.M. Wee, Y. Daryanto, "Big data analytics in supply chain management between 2010 and 2016: Insights to industries", Computer \& Industrial Engineering, vol. 115, pp. 319-330, 2018.
[3] B. Schmidt, L. Wang, D. Galar, "Semantic framework for predictive maintenance in a cloud environment", 10th CIRP Conference on Intelligent Computation in Manufacturing Engineering -CIRP ICME '16, vol. 62, pp. 583-588, 2017.

[4] European Commission, "Communication From The Commission To The European Parliament, The Council, The European Economic And Social Committee And The Committee Of The Regions A Digital Agenda for Europe", /* COM/2010/0245 f/2 */, 2010.

[5] C.A. Ardagna, V. Bellandi, P. Ceravolo, E. Damiani, M. Bezzi, C. Hebert, "A model-driven methodology for big data analytics-as-aservice", 2017 IEEE $6^{\text {th }}$ International Congress on Big Data, p. 105112, 2017.

[6] C. Colema, S. Damodaran, M. Chandramouli, E. Deuel, "Making maintenance smarter. Predictive maintenance and the digital suppy network", Deloitte University Press, May 2017.

[7] N. Amruthnath, T. Gupta, "A Research Study on Unsupervised Machine Learning Algorithms for Early Fault Detection in Predictive Maintenance", $20185^{\text {th }}$ International Conference on Industrial Engineering and Application (ICIEA), Singapore, pp. 355-361, 2018.

[8] V. Chandola, A. Banerjee, V. Kumar, "Anomaly detection: A survey", ACM Computing Surveys Journal, Vol. 41, Issue 3, Article n. 15, 2009.

[9] J.A. Harding., M. Shahbaz Srinivas, A. Kusiak, "Data Mining in Manufacturing: A Review", Journal of Manufacturing Science and Engineering,Vol. 128, No. 4, pp. 969-976 2006.

[10] A. Crespino, C. Di Biccari, M. Lezzi, M. Lazoi, "Fault Prediction in Aerospace Product Manufacturing: Development of a Model-Based Big Data Analytics Service", Workshop "BD4EI 2018: Industrial Big Data Platforms Enabling Enterprise Interoperability for Smart Services" during the IESA Conference 2018, Berlin, March 20-21, 2018. 